\title{
An orientation illusion analog to the rod and frame: Relational effects in the magnitude of the distortion
}

\author{
STANLEY COREN and VIRGIL S. HOY \\ University of British Columbia, Vancouver, British Columbia, Canada
}

\begin{abstract}
Using a printed configuration of a rod enclosed by a tilted frame, an orientation illusion analog to the classical effect was obtained. In the first experiment it was shown that the distortion obtained in this array varies in direction and magnitude as a function of degree of frame tilt, in a manner that is globally similar to that usually obtained in more traditional methods of measurement. The second experiment indicates that relational factors determine the magnitude of the effect, with increasing rod and frame separation resulting in diminished apparent tilt of the rod. Possible interactions between such an orientation illusion effect and visual-vestibular contributions to the rod and frame are discussed.
\end{abstract}

The rod-and-frame effect is a well-known phenomenon in which a line or rod surrounded by a tilted frame undergoes a shift in apparent orientation (Witkin \& Asch, 1948). There are two general classes of explanation that have been offered for the occurrence of this effect. The first views the effect as a visual-vestibular interaction; the second views it as a purely visual interaction analogous to many angular illusions.

The visual-vestibular interaction explanations for this effect depend upon fairly automatic processes that are free of cognitive mediation (cf. Howard, 1982, for a review). In general, this type of theory suggests that peripheral receptive fields may be assumed to process the image of the tilted frame, and that these in turn stimulate static orientation detectors (presumably at the vestibular nucleus). These then instigate a sequence of events which eventually produces the perception of inclination in a physically vertical line (Daunton \& Thomsen, 1979; Dichgans \& Brandt, 1978; Ebenholtz \& Glaser, 1982; Ebenholtz \& Utrie, 1983; Waespe \& Henn, 1977). However, although vestibular factors may play an important role in the formation of the percept, there appears to be a more purely visual component present. This has been demonstrated by Rock (1966), who was able to obtain the rodand-frame illusion with supine observers, a manipulation that eliminates vestibular mechanisms as the sole determinant of the effect.

In contrast, explanations based on visual interactions view the rod-and-frame illusion as analogous to many other visual-geometric illusions of orientation, such as the Zöllner or the Lipps illusions (Coren \& Girgus, 1978a). In these explanations it is the relationship between the con-

This research was supported, in part, by grants from the National Science and Engineering Research Council of Canada. The authors" mailing address is: Department of Psychology, University of British Columbia, Vancouver, British Columbia, Canada V6T IW5. tours making up the frame and the contour of the central rod which induce the tilt effect (Goodenough, Oltman, Sigman, Rosso, \& Mertz, 1979), operating via the usual mechanisms that cause illusions of direction or via some sort of cognitive inference (DiLorenzo \& Rock, 1982). Supportive evidence seems to be available for this purely visual interactive view from reports that the illusory effect diminishes with increasing separation between the test line and the inducing frame (Gogel \& Newton, 1975; Sulzer \& Zener, 1953). Other reports, using fairly large visual angle displays, have failed to find such effects (Ebenholtz, 1985). The fact that the pattern of results differs as a function of frame size has led some investigators to suggest that the visual-vestibular component is dominant for large-visual-angle displays, whereas the visual orientation component is dominant in smaller displays (Ebenholtz, 1977; Ebenholtz \& Glaser, 1982; Wenderoth, 1974).

If we, for the sake of argument, assume that at least one component of the rod-and-frame illusion is simply an illusion of direction, and that it shares common causal mechanisms with other illusions of direction (such as those isolated by Coren, Girgus, Erlichman, \& Hakstian, 1976), then the standard form of the rod-and-frame display, which pits judgments of gravitational vertical against the visual frame of reference implied by the surrounding square or rectangular frame, is not critical for the appearance of the orientation illusion. One could, for instance, suggest a simple visual array, consisting of a tilted frame with an included test line, that could be printed on a flat page, and then predict that the apparent orientation of the test line ought to be influenced by the inclination of the frame, even in the absence of visual visual-vestibular conflict, due to judgments of the interaction of gravitational vertical with the visual display. To see if such a visual array is capable of producing an analog to the rod-andframe illusion, the following experiment was conducted. 


\section{EXPERIMENT 1}

\section{Method}

All stimuli were printed in black and centered upon sheets of white paper $(21.6 \times 27.8 \mathrm{~cm})$. Each stimulus consisted of a square frame and a centered line, which served as the "rod." At normal reading distance $(40 \mathrm{~cm})$, the width of the lines making up the frame subtended a visual angle of $24^{\prime}$ and the frame was a $6^{\circ}$ square. The rod was $4^{\circ}$ long and $6^{\prime}$ wide. The frames could be presented at a $0^{\circ}, 15^{\circ}, 30^{\circ}, 60^{\circ}$, or $75^{\circ}$ clockwise tilt relative to the upright side of the stimulus sheet. Rods could be presented at any one of nine different tilts, ranging from $-4^{\circ}$ to $+4^{\circ}$ from the vertical $1^{\circ}$ steps. Taken together, these stimulus parameters produced a set of 45 discrete stimuli which were then presented in mixed order.

The subjects were 26 volunteers, 17 male and 9 female, with a mean age of 20 and vision that was normal or corrected to normal. All were naive as to the purpose of the experiment.

The subject was seated at a table and instructed to sit squarely relative to table edge and not to tilt his or her head while making judgments. The set of stimuli was presented horizontally on the surface in front of the observer, with the lower edge of the stimulus page flush against the edge of the table. The subject judged each stimulus combination separately in a forced-choice procedure by indicating whether the rod appeared to be inclined clockwise or counterclockwise. The subject then marked his or her choice on a separate answer sheet. The instructions stressed that the magnitude of inclination to be expected would be slight.

\section{Results and Discussion}

The results were analyzed using a standard Guttman (1950) scaling procedure. The results obtained via this method of rod-and-frame illusion measurement were quite stable, with a coefficient of reproducibility of $98 \%$.

The results of this experiment are shown in Figure 1. As can be seen from this figure, there are systematic variations in the apparent vertical obtained from this penciland-paper analog to rod-and-frame testing, which globally resemble the measures usually obtained from tests using apparatus in which the orientation of the test line is continuously variable and the luminous displays are presented in dark surrounds (see Howard, 1982, or Wenderoth,

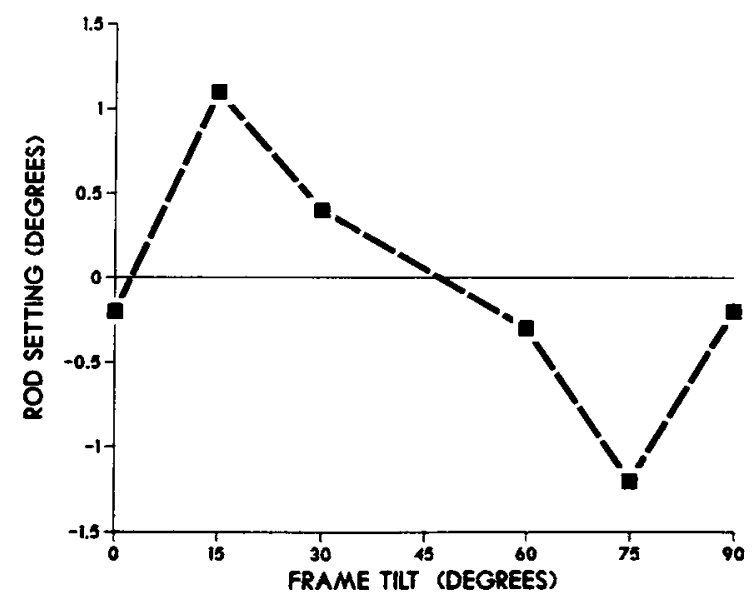

Figure 1. The judged tilt of the rod plotted as a function of the degree of tilt of the frame. Frame tilt is plotted as clockwise rotation in degrees. For rod tilts, positive numbers represent degrees clockwise and negative numbers represent degrees counterclockwise.
1977 , for reviews). The variation of apparent rod inclination as a function of frame tilt is statistically significant $[F(4,31)=14.97, p<.001]$. The magnitude of the obtained effects seems somewhat small, although small frames have been shown to produce effects in this range (e.g., Wenderoth, 1974). The nonmonotonic trend reported by Beh, Wenderoth, and Purcell (1971) for frame tilts between $15^{\circ}$ and $30^{\circ}$ does not manifest itself in these data, but depending upon specific measurement parameters and procedures, this effect often does not appear in rod-and-frame illusion data (Goodenough et al., 1979; Wenderoth, 1973). Thus, the overall pattern of results is similar to that frequently reported in the literature.

There are both methodological and theoretical consequences of these findings. To begin with, we clearly find that, using techniques similar to those commonly used for many tilt illusions, we can measure a variation in apparent tilt which seems to share many properties of the classically obtained rod-and-frame effect. Second, these effects do not seem to be easily explainable by arguments that rod-and-frame interactions involve conflicts between visual and gravitational factors, such as the ones we reviewed above, but, rather, seem to derive solely from interactions among visual components. It is thus tempting to ascribe these obtained visual tilt effects to some form of relational interaction among elements of differing spatial orientations, much like the Zöllner, Orbison, or Lipps illusions (see Coren \& Girgus, 1978a). If this is the case, then increasing the spatial separation between the test element (the rod) and the inducing elements (the frame) ought to reduce the magnitude of the obtained illusion, at least for this analog to the rod-and-frame configuration. To test this possibility, Experiment 2 was conducted.

\section{EXPERIMENT 2}

In this experiment, the relative separation between the rod and the tilt-inducing frame is varied. Ebenholtz (1985) has already shown that when one is dealing with large visual angle displays $\left(35^{\circ}\right.$ or larger), variations in rod length have little ability to vary the tilt effect. Given the actual lengths of the rods used by Ebenholtz $\left(7.6^{\circ}, 15^{\circ}\right.$, and $29.6^{\circ}$, respectively), the spatial gaps between the rod and the frame still would be some $5^{\circ}$ of visual arc or more at the smallest. Such large distances are generally ineffective for most tilt effects due to contour or relational interactions in standard illusion displays. Hence, if we are to analyze this effect as if it were a variant of a visualgeometric illusion of orientation, we must confine our attention to relational variations in smaller arrays and smaller distances between the rod and the frame. This is done in Experiment 2.

\section{Method}

Stimuli were prepared as in Experiment 1, but only two frame inclinations $\left(15^{\circ}\right.$ and $75^{\circ}$ clockwise) were utilized. The length of the rod was kept constant, at $4^{\circ}$ of arc, but the frame size could take any one of the three values, $6^{\circ}, 10^{\circ}$, or $15^{\circ}$ of visual angle 
for each side of the tilt-inducing square. As in the previous experiment, rod orientations ranged from $-4^{\circ}$ to $+4^{\circ}$ from vertical in $1^{\circ}$ increments. All permutations of frame size and rod inclination were prepared, resulting in a set of 54 discrete stimuli.

Forty-four student volunteers, 15 male and 29 female, with a mean age of 20 years and normal or corrected-to-normal vision, served as subjects. All were naive as to the purpose of the experiment. The measurement procedures were identical to those in Experiment 1 .

\section{Results and Discussion}

As in the first experiment, Guttman scaling procedures were employed. Since the measurement procedures were the same, it is not surprising that the reproducibility coefficient was virtually the same $(98 \%)$ as that found previously.

The data from this experiment are shown as Figure 2. As can be seen from this figure, there is a strong interaction between the orientation of the frame and its inclination. This effect is quite significant $[F(2,16)=37.80$, $p<.001]$. As one would expect, given the results of Experiment 1 plotted in Figure 1, one aspect of this interaction is simply the effects of the degree of tilt of the frame that affects the direction of apparent tilt of the line; the other component of this interaction, however, is due to the effect of the frame size upon the magnitude of the tilt effect obtained.

Figure 2 suggests that as the frame size becomes larger, and hence, of course, as the distance between the rod and frame increases, the ability of the frame to induce an apparent tilt of the rod diminishes. This can be statistically tested by combining the results from the two orientations, recoding them in terms of deviation from the vertical, and coding responses in the expected direction as positive and those opposite the classically expected direction as negative. Using such a procedure for the collapsing of the two frame orientations, we can then assess the effect of the distance between rod and frame on the magnitude of the

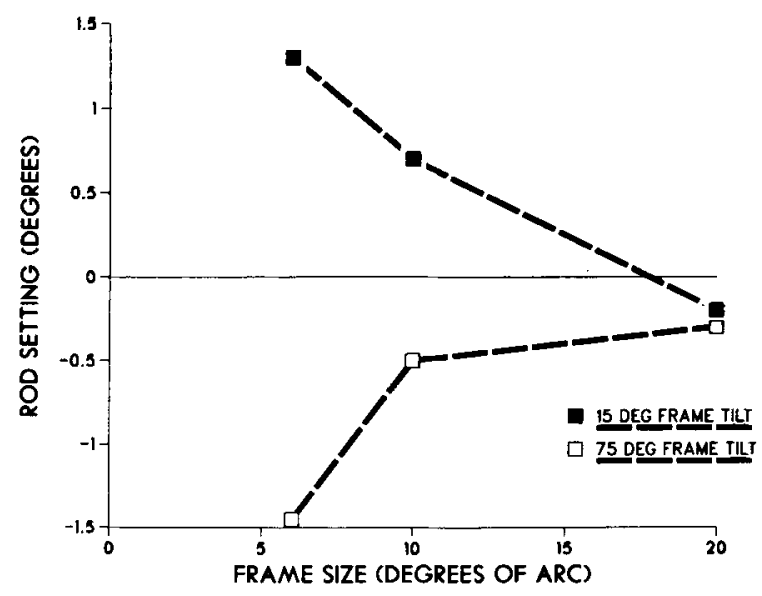

Figure 2. The judged tilt of the rod plotted as a function of the size of the frame in degrees of arc for $15^{\circ}$ and $75^{\circ}$ of frame tilt plotted separately. For rod tilts, positive numbers represent degrees clockwise and negative numbers represent degrees counterclockwise. obtained tilt, regardless of the direction of frame orientation. For the $6^{\circ}$ frame, the mean deviation from vertical observed in the rod is $1.37^{\circ}$; for the $10^{\circ}$ frame, this drops to an induced inclination of $0.58^{\circ}$. For the largest $\left(20^{\circ}\right)$ frame, the induced tilt is a negligible $0.08^{\circ}$. An analysis of variance on these collapsed measures shows that this reduction of illusion with increasing frame size is highly significant $[F(2,16)=36.30, p<.001]$.

These results, then, seem to be most clearly interpretable in terms of a relational component in the rod-andframe illusion. As the distance between the test rod and the inducing frame increases, these relational factors become less effective, hence decreasing the size of the effects observed.

\section{GENERAL DISCUSSION}

There are several conclusions which emerge from this pair of experiments, and they have both theoretical and methodological implications. To begin with, by beginning with the presumption that at least part of the obtained tilt effect observed in the rod and frame illusion is due to a visual illusion of orientation, rather than a visualvestibular conflict, a new format of presentation was developed. When simply presenting the configuration in a printed format, rather than as an illuminated configuration in dark, the effect is still obtained. Furthermore, as illustrated by the results of Experiment 1, this format produced systematic variations in the magnitude of the obtained distortion, with variations in frame inclination, which are globally analogous to those obtained in the more traditional presentation paradigm. This format is much like those used in other visual illusion studies, and, like many of these illusion presentation schemes, is suitable for group presentation with pencil-and-paper responses (see Coren \& Girgus, 1972; Coren \& Porac, 1979).

Continuing to view the distortion as an illusion of orientation allowed us to predict systematic variation in the magnitude of the effect as a function of the distance between inducing components and test element, as occurs in most classical visual-geometric illusions of orientation (see Coren \& Girgus, 1978a, for a review). As such an analysis would predict, Experiment 2 showed that the magnitude of the distortion diminished as the separation between test and inducing components increased. This clearly signifies a relational component in the illusory effect.

Given the results we have found, one must then ask how these findings relate to others reported previously. In some respects, the data pertaining to the distance between the test line and the inducing frame may be explicable by Gogel's (1978) adjacency principle. Gogel and Newton (1975) have shown that separating the inducing frame from the test line in depth tends to diminish the size of the illusory tilt. Similar effects have been found when dealing with more traditional visual geometric illusions, such as the Ponzo (Gogel, 1975), as well. Tests of the 
effect of separation in depth, however, do provide some clue as to how some of the apparent contradictions in the rod-and-frame literature may be resolved. For instance, consider the apparent lack of agreement between the results reported here and those of Ebenholtz (1985), who finds no effect of relational variation between rod and frame. Such consistency cannot be achieved if one insists that a single explanation must suffice for the full magnitude of the rod-and-frame effect. On the other hand, if we continue to analyze this distortion as a visual illusion, and recognize that most such illusions seem to come about due to the operation of several levels of processing and contributing mechanisms, as has been amply shown by previous research and analysis (e.g., Coren \& Girgus, 1978a, 1978b; Coren et al., 1976; Coren \& Porac, 1983; Coren \& Ward, 1979), some suggestions clearly seem to emerge. To begin with, it is possible that mechanisms differ for large versus small angle visual arrays. Since relational effects do not seem to play a role in large-angle configurations, it may well be that these effects may be due to visual-vestibular effects. Certainly, a large-scale array, with elements that extend well into the periphery, is more suited to the elicitation of vestibular interactions, as is informally shown in the way that large wrap-around cinema screens produce more effective apparent movement and inclination effects than do smaller (e.g., video) screens (cf. Dichgans \& Brandt, 1978). Once these visualvestibular mechanisms are evoked, the relational factors among stimulus components may well be irrelevant. This may be contrasted to the small-visual-angle arrays, where the major effects may be due to contour interactions and context effects. Data supporting this notion may be found in Ebenholtz and Glaser (1982), who report reduction of the rod-and-frame illusion as a function of separation of the elements in depth only for displays of small visual angle. In the configurations used in the experiments described above, the relational factors would be expected to predominate due to the relatively small retinal size of the stimuli employed. At intermediate sizes, both visual and nonvisual factors may be in effect. It is possible that the many conflicting reports of the pattern of variation within the rod and frame may be due to differential magnitude of contribution of the visual-vestibular and the orientation illusion components in specific configurations of the display used by various researchers. Careful consideration of parametric variations of the rod-and-frame illusion as a function of overall stimulus display size may well help to resolve some of these apparent contradictions.

At the very least, the studies presented here introduce a visual distortion which seems to be analogous to the rodand-frame effect. Although providing data which are globally similar to the classical effects obtained for the rod and frame, they seem to be more analogous to a traditional visual-geometric illusion of orientation, and seem to be strongly affected by relational factors rather than visual-vestibular interactions for the stimulus values investigated here. Furthermore, these effects are measur- able in simple printed rod-and-frame arrays which seem to be independent of a gravitational component.

\section{REFERENCES}

Beh, H. C., Wenderoth, P. M., \& Purcell, A. T. (1971). The angular function of a rod-and-frame illusion. Perception \& Psychophysics, 9(4), 353-355.

Coren, S., \& GiRGUS, J. S. (1972). A comparison of five methods of illusion measurement. Behavior Research Methods \& Instrumentation, 4, 240-244.

COREN, S., \& Girgus, J. S. (1978a). Seeing is deceiving: The psychology of visual illusions. Hillsdale, NJ: Erlbaum.

CorEN, S., \& Girgus, J. S. (1978b). Visual illusions. In H. Leibowitz \& H.-L. Teuber (Eds.), Handbook of sensory physiology: Vol. VIII. Perception (pp. 549-568). Berlin: Springer-Verlag.

Coren, S., Girgus, J. S., Ehrlichman, H., \& Hakstian, A. R. (1976). An empirical taxonomy of visual illusions. Perception \& Psychophysics, 20, 129-137.

COREN, S., \& PORAC, C. (1979). Techniques for the measurement of the Poggendorff and Zollner illusions in large group situations. Bulletin of the Psychonomic Society, 13, 219-222.

Coren, S., \& Porac, C. (1983). The creation and reversal of the Mueller-Lyer illusion through attentional manipulation. Perception, 12, 49-54.

COREN, S., \& WARD, L. M. (1979). Levels of processing in visual illusions: The combination and interaction of distortion-producing mechanisms. Journal of Experimental Psychology: Human Perception \& Performance, 5, 324-335.

Daunton, N., \& Thomsen, D. (1979). Visual modulation of otolith units in cat vestibular nuclei. Experimental Brain Research, 37, 173-176.

Dichgans, J., \& BRANDT, T. (1978). Visual-vestibular interactions: Effects on self-motion perception and postural control. In R. Held, $H$. Leibowitz, and H.-L. Teuber (Eds.), Handbook of sensory physiology: Vol. VIII. Perception. New York: Springer-Verlag.

DiLorenzo, J. R., \& Rock, I. (1982). The rod-and-frame effect as a function of the righting of the frame. Journal of Experimental Psychology: Human Perception \& Performance, 8, 536-546.

EBENholTZ, S. M. (1977). Determinants of the rod and frame effect: The role of retinal size. Perception \& Psychophysics, 22, 531-538. EaENHOLTZ, S. M. (1985). Absence of relational determination in the rod-and-frame effect. Perception \& Psychophysics, 37, 303-306.

Ebenholtz, S. M., \& Glaser, G. W. (1982). Absence of depth processing in the large-frame rod-and-frame effect. Perception \& Psychophysics, 32, 134-140.

Eaenholtz, S. M., \& UTrie, J. W., JR. (1983). Peripheral circular contours inhibit the visual orientation control system. Aviation Space \& Environmental Medicine, 54, 343-346.

Gogel, W. C. (1975). Depth adjacency and the Ponzo illusion. Perception \& Psychophysics, 17, 125-132.

GoGEl, W. C. (1978). The adjacency principle in visual perception. Scientific American, 238, 126-139.

Gogel, W. C., \& Newton, R. E. (1975). Depth adjacency and the rod-and-frame illusion. Perception \& Psychophysics, 18, 163-171.

Goodenough, D. R., Oltman, P. K., Sigman, E., Rosso, J., \& Mertz, H. (1979). Orientation contrast effects in the rod-and-frame test. Perception \& Psychophysics, 25. 419-424.

GutTMan, L. (1950). The basis for scalogram analysis. In S. A. Stouffer (Ed.), Measurement and prediction (Vol. 4, pp. 60-90). Princeton: Princeton University Press.

HowARD, I. P. (1982). Human visual orientation. New York: Wiley. Rock, I. (1966). The nature of perceptual adaptation. New York: Basic Books.

Sulzer, R. L., \& ZENER, K. E. (1953). A quantitative analysis of relations between stimulus determinants and sensitivity of the visual perception of parallelness. American Psychologist, 8, 444.

WAESPE, W., \& HENN, V. (1977). Neuronal activity in the vestibular 
nuclei of the alert monkey during vestibular and optokinetic stimulation. Experimental Brain Research, 27, 523-538.

WENDEROTH, P. M. (1973). The effects of tilted outline frames and intersecting line patterns on judgments of vertical. Perception \& Psychophysics, 14, 242-248.

WENDEROTH, P. M. (1974). The distinction between the rod-and-frame illusion and the rod-and-frame test. Perception, 3, 205-212.

Wenderoth, P. M. (1977). An analysis of the rod-and-frame illusion and its variants. In R. H. Day \& G. V. Stanley (Eds.), Studies in perception (pp. 95-141). Nedlands: University of Western Australia Press.

WITKIN, H. A., \& AsCH, S. E. (1948). Studies in space orientation: IV. Further experiments on perception of the upright with displaced visual fields. Journal of Experimental Psychology, 38, 762-782.

(Manuscript received October 17, 1985; revision accepted for publication February 26, 1986.) 